\title{
Dapsone: A Century-Old Medicine and a New Hope for Severe COVID-19
}

\author{
Vu Thuong Nguyen1, Lawrence S. Chan ${ }^{2,3^{*}}$ \\ ${ }^{1}$ Advanced Cosmeceuticals Enterprises, Inc., Sacramento, California, USA \\ ${ }^{2}$ Department of Dermatology, University of Illinois, Chicago, Illinois, USA \\ ${ }^{3}$ Department of Medicine, Captain James Lovell FHCC, North Chicago, IL, USA \\ Email: Vu.dalat79@yahoo.com, *larrycha@uic.edu
}

How to cite this paper: Nguyen, V.T. and Chan, L.S. (2022) Dapsone: A Century-Old Medicine and a New Hope for Severe COVID-19. Advances in Infectious Diseas$e s, 12,50-56$.

https://doi.org/10.4236/aid.2022.121004

Received: December 22, 2021

Accepted: February 8, 2022

Published: February 11, 2022

Copyright $\odot 2022$ by author(s) and Scientific Research Publishing Inc. This work is licensed under the Creative Commons Attribution International License (CC BY 4.0).

http://creativecommons.org/licenses/by/4.0/

(c) (i) Open Access

\begin{abstract}
Background: The 2020 pandemic of COVID-19 infection, which has already lasted more than 22 months, is not subsiding. Despite the available vaccinations, the infection is still claiming many lives throughout the world, especially among the unvaccinated people. The newly discovered and fast spreading Omicron variant also raises the concern if vaccination alone will be sufficient to win this battle. Purpose: In this concept paper, we examine the possible use dapsone, a century-old and safe medication, to treat patients with severe cases of pulmonary COVID-19 infection. Methods: We aim to delineate the similarities between IgA-mediated neutrophil-directed blistering skin diseases and the severe pulmonary COVID-19 infection by reviewing the relevant medical literatures, in order to compare the pathological mechanisms of these two different clinical situations. Results: Analysis of published reports reveals strong evidence pointing to the involvement of IgA and neutrophils in both clinical situations. In addition, results of a small clinical trial showed efficacy of dapsone in patients suffered with severe COVID-19 infection. Conclusions: Since dapsone is effective in treating IgA-mediated skin diseases by blocking the binding of neutrophils to the tissue site, it could also be an effective treatment for severe cases of COVID-19 lung injury, which has a similar pathological mechanism.
\end{abstract}

\section{Keywords}

Dapsone, IgA, Neutrophils, COVID-19, Lung Injury

\section{Introduction}

The COVID-19 pandemic has been upon us for more than 22 months. Despite the availability of vaccination, the infection still claims many lives, especially the 
unvaccinated people. The ability of virus to mutate and evade immune defense prompts medical community to seek other means to win this battle. In this short paper, we examine the rationale and the possibility of utilizing a century-old medication dapsone to help COVID-19 patients with critical lung injury. We will first delineate the established knowledge of the therapeutic effects and the molecular mechanism of dapsone on skin diseases. After comparing the pathological mechanisms between skin disease and COVID-19-related lung injury, we will then discuss the proposed treatment.

\section{Dapsone Treatment for IgA-Mediated Skin Diseases}

Dapsone, a sulfone class of medication developed in 1908, has been extensively and confidently utilized by dermatology physicians over a century. Initially used as an antibiotic for leprosy, dapsone has been subsequently adapted to treat several skin diseases that are pathologically characterized by tissue deposition of immunoglobulin A (IgA) and infiltration of one type of white blood cells termed neutrophils. Dermatitis herpetiformis, an intensively itching and blistering skin disease related to gluten-sensitive enteropathy, is such an excellent example of IgA-mediated neutrophil-directed skin disease highly responsive to dapsone treatment [1]. Autoimmune linear IgA bullous dermatosis and IgA pemphigus are two other good examples [2] [3]. The rapid resolution of this group of skin diseases, which could occur as fast as within 1 - 3 days of dapsone treatment, is well documented. In fact, dapsone is considered a first-line medication for these IgA-mediated skin diseases [1] [2].

The medical community recognized not only the clinical usefulness of dapsone for the treatment of IgA-mediated neutrophil-directed skin diseases, but also delineated the mechanism of how dapsone stops the destructive effects of neutrophils. Research conducted in our laboratory and in others has demonstrated that dapsone inhibits the binding of neutrophils to IgA. Since the adherence to IgA by neutrophils is a prerequisite for their activation that subsequently leads to tissue destruction, dapsone thus prevents tissue destruction by blocking the neutrophil binding to IgA on the target tissue, in addition to other anti-inflammatory effects [1] [4] [5] [6].

Specifically, in our previous study on the mechanism of dapsone in inhibiting neutrophil binding of skin we utilized a modified neutrophil adherence assay. We designed an IgA complex model with IgA binding to skin basement membrane zone (BMZ) antigen. This IgA complex model system allowed us to examine the effects of neutrophil chemokine GM-CSF and dapsone on the binding of neutrophils to the IgA complex. Incubating IgA-BMZ antigen complexes with dapsone prior to exposing them to neutrophils revealed that dapsone inhibited neutrophil adherence by direct impact on IgA rather than cytotoxic effect on neutrophils [5]. On the molecular level, dapsone, with its unique chemical structure diaminodiphenyl sulfone, could bind to the IgA protein and interfere the IgA's Fc portion, the binding site for neutrophils, so that IgA could no long- 
er interact with the Fc receptor (FcaRI) of neutrophils. This could explain the rapid effect of dapsone in the treatment of IgA-mediated neutrophil-directed skin diseases. The molecular mechanism regarding IgA and its interaction with FcaRI on inflammatory cells in diseases has been delineated extensively [6]. The interference of dapsone to neutrophil adherence to IgA immune complex, either by inducing some conformational changes or by steric hindrance on the $\mathrm{Fc}$ of IgA, could add a new therapeutic opportunity for other pathological conditions where IgA immune complex also plays a critical role.

\section{Similarities between IgA-Mediated Skin Diseases and COVID-19 Lung Injuries}

Now what is the link between IgA-mediated skin diseases and COVID-19 infection? This link is based on their similar underlying pathological mechanisms. While the majority cases of COVID-19 (SARS-CoV-2) infection have mild symptom or asymptomatic, severe cases that occur in less than $15 \%$ of infection, manifest with a surge of proinflammatory proteins termed cytokines ("cytokine storm") and neutrophil infiltration, and lung tissue destruction and other organ failures [7] [8]. This lung injury event, as it turns out, is likely mediated by IgA-driven neutrophil activation. The evidence supporting this mechanism are illustrated below. Early antibody response in severe COVID-19 infection is characterized by a surge of IgA, rather than the typical response of IgM. The ratios of IgA to IgG were markedly higher in severe cases compared to milder cases. Moreover, serum IgA levels parallel the lung injury severity. Particularly, the level of SARS-CoV-2-specific IgA antibodies, but not IgG or IgM antibodies, significantly correlated with disease severity [9] [10]. Studies on autopsies of COVID-19 victims showed that SARS-CoV-2 is found in both alveola epithelial cells type 1 and type 2 [11]. High level of both dimeric and monomeric IgA antibodies against SARS-CoV-2 is found in respiratory mucosa alveola [12]. Many medical literatures reported that COVID-19-infected patients developed IgA-mediated blood vessel injury (IgA-vasculitis) or IgA-mediated kidney injury (IgA-nephropathy) with documented IgA deposits in their respective organs blood vessels or kidney [13]. In addition, the increase of blood and lung fluid levels of neutrophils in COVID-19 patients parallels the progression of their disease severity. Furthermore, prominent neutrophil infiltration and adherence to alveola epithelial cells with alveolar wall injury, necrosis, and microthrombi in the patients' lung, not associated with superinfection or mechanical ventilation, were observed only in a subset of COVID-19 patients who had rapid disease progression and severe respiratory failure [14].

\section{A Treatment Proposal}

Since the mechanism of skin injury by IgA-mediated neutrophil-directed skin disease is significantly similar to that of lung injury by COVID-19 infection (Table 1), the logical question will be why not use the medication proven useful for 
Table 1. Similarities between IgA-mediated neutrophil-directed skin diseases and the severe COVID-19 lung injury.

\begin{tabular}{|c|c|c|}
\hline Disease Characteristics & $\begin{array}{l}\text { IgA-mediated Neutrophil-directed } \\
\text { Skin Diseases }\end{array}$ & $\begin{array}{c}\text { Severe COVID-19 Infection with Lung Tissue } \\
\text { Injuries }\end{array}$ \\
\hline Circulating IgA & $\begin{array}{l}\text { Skin-specific IgA are detected in linear } \\
\text { IgA dermatosis and IgA pemphigus. }\end{array}$ & $\begin{array}{l}\text { Early immunoglobulin surge is IgA, } \\
\text { rather than IgM. } \\
\text { IgA: IgM ratios markedly increase } \\
\text { compared to milder cases. } \\
\text { IgA levels parallel disease severity. } \\
\text { COVID-19-specific IgA levels significantly } \\
\text { correlate with disease severity. }\end{array}$ \\
\hline Tissue-bound IgA & $\begin{array}{l}\text { Skin tissue IgA deposits are detected in } \\
\text { linear IgA dermatosis, IgA pemphigus, } \\
\text { and dermatitis herpetiformis. }\end{array}$ & $\begin{array}{l}\text { IgA-vasculitis (with IgA deposited in blood } \\
\text { vessels) occurred in some COVID-19 patients. } \\
\text { IgA-nephropathy (with IgA deposited in } \\
\text { kidney) occurred in some COVID-19 patients. } \\
\text { Both dimeric IgA and monomeric IgA against } \\
\text { SARS-CoV-2 in respiratory mucosa alveola were } \\
\text { detected in severe COVID patients. }\end{array}$ \\
\hline Neutrophil Tissue Infiltration & $\begin{array}{l}\text { Neutrophil infiltration in skin is } \\
\text { detected in linear IgA dermatosis, } \\
\text { IgA pemphigus, and dermatitis } \\
\text { herpetiformis. }\end{array}$ & $\begin{array}{l}\text { Neutrophil infiltration in lung tissues is } \\
\text { primarily detected in severe cases. } \\
\text { Neutrophils adhered to alveola epithelial cells } \\
\text { were found in autopsy of COVID patients. }\end{array}$ \\
\hline Response to dapsone treatment & $\begin{array}{l}\text { Responses are good to excellent. } \\
\text { Rapid resolution of skin diseases can } \\
\text { be achieved as early as one to three } \\
\text { days. }\end{array}$ & $\begin{array}{l}\text { A small study of } 44 \text { ICU-admitted severe } \\
\text { COVID- } 19 \text { patients documented } 100 \% \text { survival } \\
(22 / 22) \text { of patients treated with dapsone, } \\
\text { compared to } 36 \% \text { mortality }(8 / 22) \text { of } \\
\text { patients who were not treated. } \\
\text { Leprosy patients infected by COVID-19 while } \\
\text { on dapsone treatment did not become severely } \\
\text { ill and recovered. }\end{array}$ \\
\hline
\end{tabular}

IgA-mediated skin diseases to treat severe COVID-19? Therefore, we propose that since dapsone could inhibit IgA-mediated neutrophil-directed skin injury, it should be tried on severe COVID-19 patients to block lung injury (Figure 1).

In fact, our proposal is supported by the results of a small study. A recent clinical trial of 44 COVID-19 patients in ICU with similar clinical condition documented that in the group of dapsone-treated patients, all 22 patients survived, whereas in the group of patients did not receive dapsone, 8 of 22 patients did not survive. Interestingly, leprosy patients who were infected with COVID-19 while on dapsone treatment did not become severely ill and recovered [15]. Thus, the news of successful short course trial in the US has been followed by two large clinical trials of dapsone for COVID-19 that are currently undergoing at the Hunt Regional Medical Center in Texas, USA and the McGill University Health Centre in Quebec, Canada, with estimated primary completion dates of December 31, 2021 and March 31, 2022, respectively. The results of these trials are not yet available as of January 5, 2022 [16] [17]. 


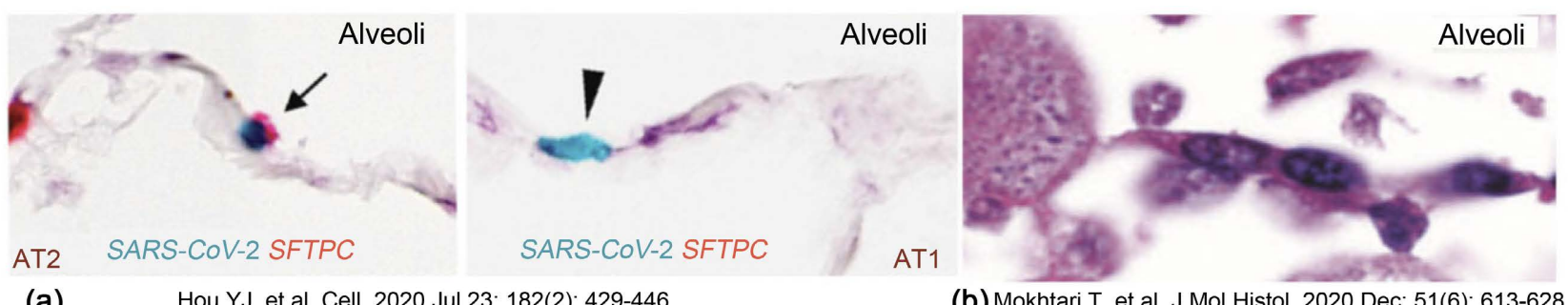

(a) Hou YJ, et al. Cell. 2020 Jul 23; 182(2): 429-446

(b) Mokhtari T, et al. J Mol Histol. 2020 Dec; 51(6): 613-628

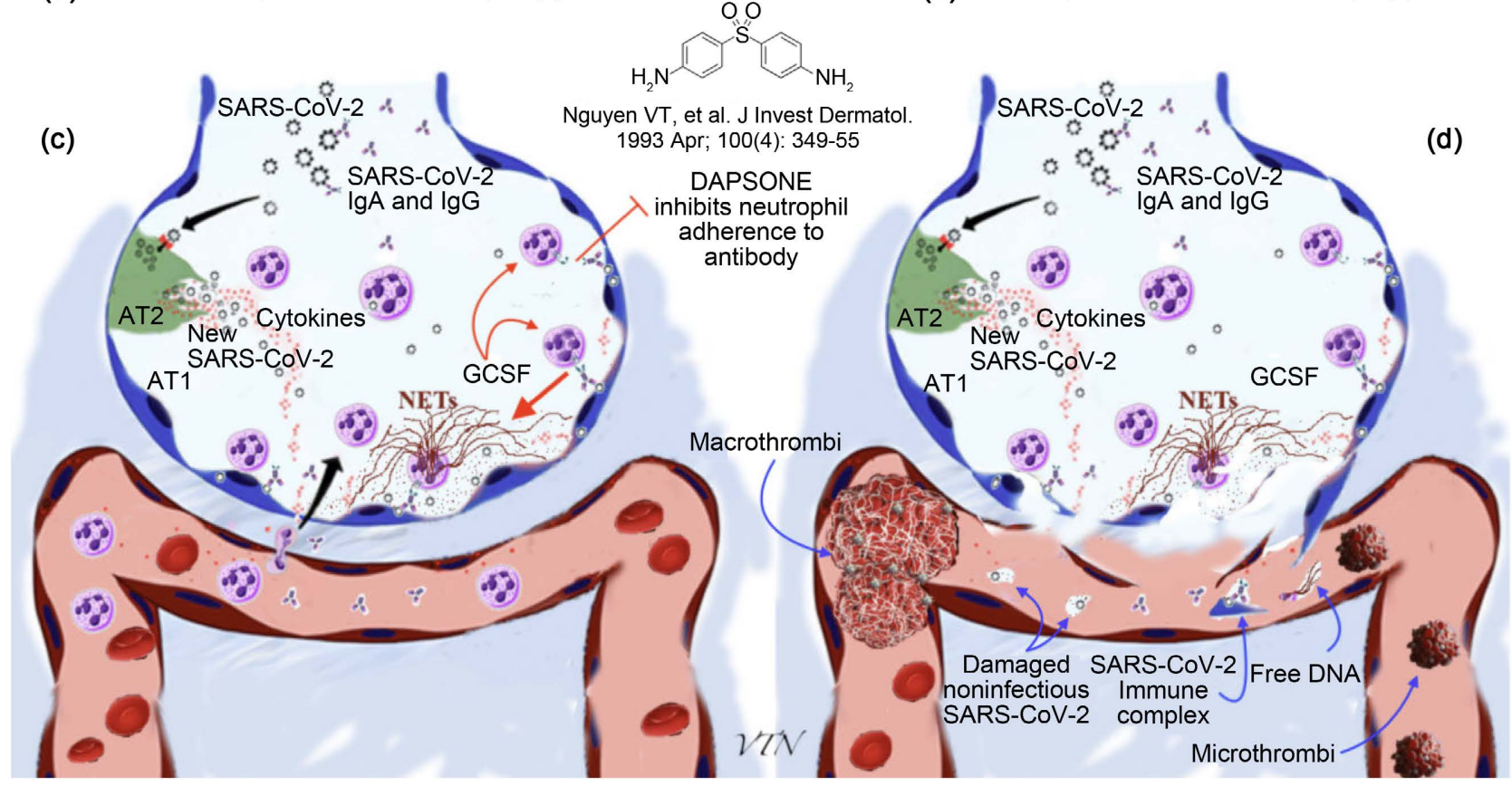

Figure 1. Illustrations of the molecular mechanism of IgA/Neutrophil destruction of pulmonary alveola and vasculature in severe COVID-19 and the proposed blocking mechanism of dapsone. A and B: evidence form autopsy of COVID-19 patients. (a). Histopathology evidence of SARS-CoV-2 infecting alveolar cells type 2 (AT2, labeled with SFTPC) and alveolar cells type 1 (AT1) (Hou YJ et al.). Correspondingly, high level of monomeric IgA and IgG against SARS-CoV-2 have been found in respiratory mucosa alveola in samples of bronchoalveolar lavage fluid from COVID-19 patients by Sterlin et al. (b). Histopathology evidence of neutrophil adhered to alveola cells by Mokhtari et al. (c). Schematic illustration of a mechanism on adherence of neutrophils to lung tissue-bound antibodies followed by activation, leading to inflammatory responses. The blocking action site of dapsone is depicted. (d). Schematic illustration of a pathological mechanism occurring in severe COVID-19 lung injury and multiorgan syndrome: In the absence of dapsone inhibition, overactive neutrophils that activated by adherence to lung tissue-bound antibodies, especially IgA, can destroy alveola as well as lung epithelial-endothelial barrier, leading to leakage of damaged noninfectious SARS-CoV-2 viral particles, SARS-CoV-2-antibody immune complex, free DNA, and other cellular debris to the circulation. Damaged endothelial cell initiates formation of macrothrombi and microthrombi that can further migrate to other organs, leading to multiorgan syndrome in severe COVID-19. Dapsone could stop the whole destructive process by inhibiting neutrophil adherence to $\operatorname{IgA}$.

After a century of usage, dapsone is generally recognized as a safe medication. Other than the rare occurrence of the following side effects (usually after a long-term usage), blood oxygen level reduction, white blood cells decrease, hypersensitivity, psychosis, and neuropathy, dapsone has a very good safety profile and is readily available and inexpensive [1]. Careful monitoring these side effects, especially for usage in a short-term basis, should be sufficient to ensure that no major problem would occur in the patients receiving dapsone. Despite the available vaccines, the current COVID-19 pandemic still claims many lives. 
According to Johns Hopkins University-COVID resource center, in October 2021 alone, there have been 198,000 COVID-19 related deaths worldwide including more than 45,000 American. The rapid rise of multiple variants of COVID-19 viruses, including the Delta variant and the new and fast spreading Omicron variant with more than 30 mutations to the spike protein, also raises the concern if vaccination alone will be sufficient to win the battle for this pandemic that has already lasted longer than 22 months [18]. While waiting for the results from the large clinical trial may give the medical community more evidence in support for dapsone therapy in the COVID-19 setting, select use of this medication, guided by clinical condition and IgA data, at this time may be called for in cases of impending respiratory compromise on an emergency basis. Specifically, we recommend that COVID-19 patients, who have severe disease and are admitted to the hospital, should be checked for their serum IgA levels. If their IgA levels are elevated, dapsone at the dose of $200 \mathrm{mg}$ per day should be administered during the acute disease phase. After the acute phase, a lower dose of dapsone (50 - $100 \mathrm{mg}$ per day) for two more weeks should be utilized to prevent relapse [15]. Saving life for patients with severe COVID-19 infection requires our urgent actions.

\section{Conflicts of Interest}

The authors declare no conflicts of interest regarding the publication of this paper.

\section{References}

[1] Wozel, G. and Blasum, C. (2014) Dapsone in Dermatology and Beyond. Archives of Dermatological Research, 306, 103-124. https://doi.org/10.1007/s00403-013-1409-7

[2] Diaz, M.S., Morita, L., Ferrari, B., Sartori, S., Greco, M.F., Sobrevias Bonells, L., et al. (2019) Linear IgA Bullous Dermatosis: A Series of 17 Cases. Actas Dermo-Sifiliográficas, 110, 673-680. https://doi.org/10.1016/j.ad.2018.06.017

[3] Wang, J., Kwon, J., Ding, X., Fairley, J.A., Woodley, D.T. and Chan, L.S. (1997) Nonsecretory IgA1 Autoantibodies Targeting Desmosomal Component Desmoglein 3 in Intraepidermal Neutrophilic IgA Dermatosis. The American Journal of Pathology, 150, 1901-1907.

[4] Hendrix, J.D., Mangum, K.L., Zone, J.J. and Gammon, W.R. (1990) Cutaneous IgA Deposits in Bullous Diseases Function as Ligands to Mediate Adherence of Activated Neutrophils. Journal of Investigative Dermatology, 94, 667-672. https://doi.org/10.1111/1523-1747.ep12876246

[5] Nguyen, V.T., Kadunce, D.P., Hendrix, J.D., Gammon, W.R. and Zone, J.J. (1993) Inhibition of Neutrophil Adherence to Antibody by Dapsone: A Possible Therapeutic Mechanism of Dapsone in the Treatment of IgA Dermatoses. Journal of Investigative Dermatology, 100, 349-355. https://doi.org/10.1111/1523-1747.ep12471811

[6] Breedveld, A. and van Egmond, M. (2019) IgA and FcaRI: Pathological Roles and Therapeutic Opportunities. Frontiers in Immunology, 10, Article No. 553. https://doi.org/10.3389/fimmu.2019.00553

[7] Tomar, B., Anders, H.J., Desai, J. and Mulay, S.R. (2020) Neutrophils and Neutro- 
phil Extracellular Traps Drive Necroinflammation in COVID-19. Cells, 9, 1383. https://doi.org/10.3390/cells9061383

[8] Mokhtari, T., Hassani, F., Ghaffari, N., Ebrahimi, B., Yarahmadi, A. and Hassanzadeh, G. (2020) COVID-19 and Multiorgan Failure: A Narrative Review on Potential Mechanisms. Journal of Molecular Histology, 51, 613-628.

https://doi.org/10.1007/s10735-020-09915-3

[9] Yu, H.Q., Sun, B.Q., Fang, Z.F., Zhao, J.C., Liu, X.Y., Li, Y.M., et al. (2020) Distinct Features of SARS-CoV-2-Specific IgA Response in COVID-19 Patients. European Respiratory Journal, 56, Article ID: 2001526.

https://doi.org/10.1183/13993003.01526-2020

[10] Hasan, A.O., Bomze, D., Risch, L., Brugger, S.D., Paprotny, M., Weber, M., et al. (2020) Severe COVID-19 Is Associated with Elevated Serum IgA and Antiphospholipid IgA-Antibodies. Clinical Infectious Diseases, 73, e2869-e2874.

https://doi.org/10.1093/cid/ciaa1496

[11] Hou, Y.J., Okuda, K., Edwards, C.E., Martinez, D.R., Asakura, T., Dinnon, K.H., et al. (2020). SARS-CoV-2 Reverse Genetics Reveals a Variable Infection Gradient in the Respiratory Tract. Cell, 182, 429-446.e14.

https://doi.org/10.1016/j.cell.2020.05.042

[12] Sterlin, D., Mathian, A., Miyara, M., Mohr, A., Anna, F., Claër, L., et al. (2020) IgA Dominates the Early Neutralizing Antibody Response to SARS-CoV-2. Science Translational Medicine, 13, eabd2223. https://doi.org/10.1101/2020.06.10.20126532

[13] Allez, M., Denis, B., Bouaziz, J.-D., Battistella, M., Zagdanski, A.-M., Bayart, J., et al. (2020) Covid-19 Related IgA Vasculitis. Arthritis Rheumatology, 72, 1952-1958. https://doi.org/10.1002/art.41428

[14] Borczuk, A.C., Salvatore, S.P., Seshan, S.V., Patel, S.S., Bussel, J.B., Mostyka, M., et al. (2020) COVID-19 Pulmomary Pathology: A Multi-Institutional Autopsy Cohort from Italy and New York City. Modern Pathology, 33, 2156-2168. https://doi.org/10.1038/s41379-020-00661-1

[15] Kanwar, B., Lee, C.J. and Lee, J.H. (2021) Specific Treatment Exists for SARS-CoV-2 ARDS. Vaccines (Basel), 9, 635. https://doi.org/10.3390/vaccines 9060635

[16] Critical Care Results of SARS-CoV-2 ARDS by Dapsone and Standard COVID-19 Treatment. Clinicaltrials.gov. https://clinicaltrials.gov/ct2/show/record/NCT04918914

[17] Dapsone Coronavirus SARS-CoV-2 Trial (DAP-CORONA) COVID-19 (DAP CORONA). Clinicaltrials.gov. https://clinicaltrials.gov/ct2/show/NCT04935476

[18] Callaway, E. (2021) Heavily Mutated Omicron Variant Puts Scientists on Alert. Nature, 600, 21. https://doi.org/10.1038/d41586-021-03552-w 